\title{
Clinical and linguistic validation of a Polish version of the Pulmonary Embolism Quality of Life Questionnaire: a disease-specific quality of life questionnaire for patients after acute pulmonary embolism
}

\author{
Jerzy Wiliński', Ositadima Chukwu², Katarzyna Ciuk², Radosław Borek', Anna Skwarek \\ $1_{1}^{\text {st }}$ Department of Internal Medicine with Cardiology Subdivision, Blessed Marta Wiecka District Hospital, Bochnia, Poland \\ ${ }^{2}$ Faculty of Medicine, Jagiellonian University Medical College, Kraków, Poland
}

\author{
Correspondence to: \\ Jerzy Wiliński, MD, PhD, \\ $1^{\text {st }}$ Department of Internal \\ Medicine with Cardiology \\ Subdivision, \\ Blessed Marta Wiecka \\ District Hospital, \\ Krakowska 31, \\ 32-700 Bochnia, Poland, \\ phone: +48146153317 \\ e-mail: \\ putamen@interia.pl \\ Copyright by the Author(s), \\ 2021 \\ Kardiol Pol. 2021; \\ 79 (9): 1019-1021; \\ DOl: 10.33963/KP.a22021.0074 \\ Received: \\ May 15, 2021 \\ Revision accepted: \\ July 23, 2021 \\ Published online: \\ July 26,2021
}

\section{INTRODUCTION}

Acute venous thromboembolism involving deep vein thrombosis and/or pulmonary embolism (PE) has a broad array of early and long-term complications including recurrent episodes, bleeding complications related to anticoagulant treatment, persisting dyspnea or poor physical capacity, post-thrombotic syndrome, and chronic thromboembolic pulmonary hypertension. The underestimated sequala is decreased quality of life (QoL) [1-3]. Questionnaires for assessing QoL after an episode of acute PE were developed in several languages, however, there is none in Polish. The Pulmonary Embolism Quality of Life Questionnaire (PEmb-QoL) fits very well in the modern approach to the holistic management of PE and was proven to be a good instrument to detect and measure the intensity of symptoms and physical functioning. PEmb-QoL was first developed in Dutch and translated into English (2009). It was later validated in several languages: French (2014), German (2015), Norwegian (2015), and Chinese (2018) [4-8]. In this paper, we report the results of the validation of this questionnaire in Polish.

\section{METHODS}

The Pulmonary Embolism Quality of Life Questionnaire

The Pulmonary Embolism Quality of Life Questionnaire questionnaire consists of 9 questions (40 items) clustered into 6 dimensions: frequency of complaints $(\mathrm{FO})-\mathrm{Q} 1$; activities of daily living limitations (AD) - Q4; work-related problems (WR) - Q5; social limitations (SL) - Q6; intensity of complaints (IO) - Q7, Q8; emotional complaints (EC) - Q9. Questions Q2 and Q3 provide descriptive information and are not used to calculate scores. Higher scores indicate a worse outcome. Questions Q1, Q4, Q5, Q9 are reversely scored. Scores of a given dimension are calculated by averaging scores of all items in that dimension. Transformed dimensions score is calculated in a two-step process. The score of each item is rescaled in such a way that 100 corresponds to a possible maximal score and 0 corresponds to a minimal score. We take a mean of items that constitute a given dimension.

We performed a forward-backward translation of the English version of the PEmb-QoL questionnaire into Polish according to previously published recommendations [9]. The final Polish version is provided in the Supplementary material.

\section{Patients sample}

The inclusion criteria included age above 18 years, the history of acute PE confirmed objectively with computed tomography angiography of pulmonary arteries, ventilation/perfusion scintigraphy, angiography of pulmonary arteries or with transthoracic or transesophageal echocardiography with the detection of thrombi in the pulmonary arteries.

The exclusion criteria were the refusal to participate in the survey, chronic thromboembolic pulmonary hypertension, severe dementia, residence in a nursing facility, problems with using the Polish language. The median time 
from an episode of $\mathrm{PE}$ to completing the questionnaire was 21 months (interquartile range [IQR], 13-31).

\section{Data collection}

The patients who were enrolled in the study were invited by a phone call to have an appointment at the cardiologist outpatient office at the hospital where they received a PEmb-QoL questionnaire and the Polish version of the 36Item Short-Form Health Survey (SF-36) [10]. Patients were asked to complete and return both questionnaires. Patients who were not willing to visit the office, were asked to complete and return both questionnaires using a pre-stamped return envelope. The same scenario was used 14 days later.

\section{Ethical issues}

The study protocol was approved by the Bioethics Committee of the Regional Medical Chamber in Tarnow, Poland (No. 3/0177/2019).

\section{Statistical analysis}

Statistical analysis was performed using R software, version 4.0.2 with the "Psych" package. All tests were two-sided with a $5 \%$ significance level.

A detailed description of the statistical analysis can be found in the supplementary material.

\section{RESULTS AND DISCUSSION}

The final sample involved 103 patients. Included patients were 67 years old (IQR 67.00-72.50). The youngest participant was 22 and the oldest one was 87 years old. Sixty-four patients $(62.14 \%)$ were aged $\geq 65$ years, $63(61.17 \%)$ of them were male. Clinical characteristics are presented in the Supplementary material, Table S1.

\section{Scores of 6 dimensions of PEmb-QoL}

The median score was 2 for FO (IQR 1.38-2.50), 1.92 for AD (IQR 1.31-2.42), 1.5 for WR (1-2), 2 for SL (IQR 1-3), 2.5 for IO (IQR 1.5-3.5), 2.3 for EC (1.5-2.95). Scores of aspects ( 0 indicates lowest possible score and 100 indicates maximal possible score) are shown in supplementary material, Figure S1.

\section{Floor and ceiling effect}

All dimensions had floor effects ranging from $8.74 \%$ for EC to $37.86 \%$ for SL, for four dimensions the floor effect was substantial. Three dimensions had a non-zero ceiling effect AD, WR, and SL with 7.77\%, 30.1\%, 6.8\%, respectively (Supplementary material, Table S2).

\section{Factor analysis}

Factor analysis supported the underlying dimensions in general. The screen test identified four factors with eigenvalues greater than $2(17.57 ; 3.26 ; 2.70 ; 2.15)$. They accounted for $29 \% ; 17 \% ; 12 \%$ and $10 \%$ of total variance. Factor 1 included items - Q4, Q6, Q8; Factor 2 included most of Q9; Factor 3 included most of Q1, Q7; Factor 4 included Q5 (Supplementary material, Table S3).

\section{Reliability and reproducibility}

A half of Cronbach's alpha coefficients were $>0.9$ except for FO 0.81; WR 0.89; IO 0.67; indicating high internal consistency. Items were positively related to each other, with all average inter-item correlations $>0.3$ (Supplementary material, Table S4). The values of item total correlations were ranging from 0.30 to 0.87 .

PEmb-QoL dimensions were mostly moderately or well correlated between themselves $(0.37 \leq r \leq 0.82)$, with the strongest correlation between the intensity of complaints and frequency of complaints (0.82) (Supplementary material, Table S5).

Intraclass correlation coefficients (ICCs) for test-retest analysis were high, ranging between 0.58 for SL (Q6) and 0.92 for FO (Q1) (Supplementary material, Table S6).

\section{Construct validity}

For correlations between aspects of PEmb-QoL and SF-36 Spearman's correlation coefficient ranged between -0.93 (for AD [Q4] and physical functioning) and -0.12 (for SL [Q6] and general health). The PEmb-QoL dimensions: AD (Q4), WR (Q5), SL (Q6), IO (Q7, Q8), EC (Q9) showed higher correlations with the SF-36 physical component summary, whereas FO (Q1) showed a higher correlation with the SF-36 mental component (Supplementary material, Table S7). Overall, these correlations supported good convergent validity.

PEmb-QoL dimension scores were mostly weakly correlated with clinical characteristics (Supplementary material, Table S8). The factor that influenced PEmb-QoL the most was the presence of cardiovascular disease. This indicates adequate discriminant validity.

\section{Floor and ceiling effects}

A substantial ceiling effect occurs only in one dimension -WR as in most previous studies [4-7]. A substantial floor effect was observed in four dimensions. As it was pointed out in the aforementioned studies [5], high floor and ceiling effects in particular aspects may be an effect of: a small number of items per dimension (Q6 - SL); a small range of possible answers per item (Q5 - WR). It is possible that the range of the scale is not large enough to accommodate the distribution of the data, or there is social desirability bias.

\section{Factor analysis}

There were different approaches to the factor analysis in the previous validations of PEmb-QoL. We chose to preserve clinical defined factors as in the primary English version. Preserving clinically defined factors had practical advantages. It makes it plausibly easier to extrapolate clinical outcomes from studies in most countries. Furthermore, it allows us to use foreign software to compute 
PEmb-QoL scores. Clinically defined dimensions have the same number of answers per item and make computing scores easier. Clinical definitions were designed to be intuitive and easy. Factor analysis performed in our study can suggest a different structure of dimensions from the one in the English version of the questionnaire.

\section{Test-retest reliability}

There are no clear guidelines about the exact duration of the time gap before reassessment when test-retest reliability is verified [11]. We chose a 2-week period to retest our patients. ICC value was low only for SL, which is similar to other studies' results. SL had the lowest ICC in those publications $[4,5]$. ICC of similar value had been accepted in previous studies [5]. All other ICC values were over 0.7. This indicates good overall reproducibility. To sum up, all exceptions to strict psychometric norms are not specific for the Polish version of this tool and were accepted in previous validations. The questionnaire has been proved to be a valid tool with adequate reliability and reproducibility.

\section{CONCLUSIONS}

The Polish version of the PEmb-QoL questionnaire is a valid tool in estimating disease-specific QoL.

\section{Supplementary material}

Supplementary material is available at https://journals. viamedica.pl/kardiologia_polska.

\section{Article information}

Conflict of interest: None declared.

Open access: This article is available in open access under Creative Common Attribution-Non-Commercial-No Derivatives 4.0 International (CC BY-NC-ND 4.0) license, allowing to download articles and share them with others as long as they credit the authors and the publisher, but without permission to change them in any way or use them commercially. For commercial use, please contact the journal office at kardiologiapolska@ptkardio.pl.

How to cite: Wiliński J, Chukwu O, Ciuk K, et al. Clinical and linguistic validation of Polish version of Pulmonary Embolism Quality of Life Questionnaire: disease-specific quality of life questionnaire for patients after acute pulmonary embolism. Kardiol Pol. 2021; 79(9): 1019-1021, doi: $10.33963 /$ KP.a2021.0074.

\section{REFERENCES}

1. Konstantinides SV, Meyer G, Becattini C, et al. The Task Force for the diagnosis and management of acute pulmonary embolism of the European Society of Cardiology (ESC). 2019 ESC Guidelines for the diagnosis and management of acute pulmonary embolism developed in collaboration with the European Respiratory Society (ERS): The Task Force for the diagnosis and management of acute pulmonary embolism of the European Society of Cardiology (ESC). Eur Respir J. 2019; 54(3): 543-603, doi: 10.1183/13993003.01647-2019, indexed in Pubmed: 31473594.

2. Roik M, Wretowski $D$, Łabyk $A$, et al. Initial experience of pulmonary embolism response team with percutaneous embolectomy in intermediate-high- and high-risk acute pulmonary embolism. Kardiol Pol. 2019; 77(2): 228-231, doi: 10.5603/KP.a2018.0239, indexed in Pubmed: 30566224.

3. Sławek-Szmyt S, Jankiewicz S, Smukowska-Gorynia A, et al. Implementation of a regional multidisciplinary pulmonary embolism response team: PERT-POZ initial 1-year experience. Kardiol Pol. 2020; 78(4): 300-310, doi: 10.33963/KP.15230, indexed in Pubmed: 32165606.

4. Rochat M, Méan M, Limacher A, et al. Quality of life after pulmonary embolism: validation of the French version of the PEmb-QoL questionnaire. Health Qual Life Outcomes. 2014; 12: 174, doi: 10.1186/s12955-014-01744, indexed in Pubmed: 25464821.

5. Frey PM, Méan M, Limacher A, et al. Quality of life after pulmonary embolism: Prospective validation of the German version of the PEmb-QoL questionnaire. Thromb Res. 2015; 135(6): 1087-1092, doi: 10.1016/j. thromres.2015.03.031, indexed in Pubmed: 25887632.

6. Klok FA, Cohn DM, Middeldorp S, et al. Quality of life after pulmonary embolism: validation of the PEmb-QoL Questionnaire. JThromb Haemost. 2010; 8(3): 523-532, doi: 10.1111/j.1538-7836.2009.03726.x, indexed in Pubmed: 20025645

7. Sun X, Li J, Shi J, et al. Validating the Chinese version of the PEmb-QoL questionnaire: A measure for quality of life assessment after pulmonary embolism. Thromb Res. 2018; 166: 86-91, doi: 10.1016/j.thromres.2018.04.020, indexed in Pubmed: 29704766.

8. Tavoly M, Jelsness-Jørgensen LP, Wik HS, et al. Quality of life after pulmonary embolism: first cross-cultural evaluation of the pulmonary embolism quality-of-life (PEmb-QoL) questionnaire in a Norwegian cohort. Qual Life Res. 2015; 24(2): 417-425, doi: 10.1007/s11136-014-0779-4, indexed in Pubmed: 25120017.

9. Beaton DE, Bombardier C, Guillemin F, et al. Guidelines for the process of cross-cultural adaptation of self-report measures. Spine (Phila Pa 1976). 2000; 25(24): 3186-3191, doi: 10.1097/00007632-200012150-00014, indexed in Pubmed: 11124735.

10. Tylka J, Piotrowicz R. Cardiac rehabilitation. Quality of life SF-36 questionnaire - the Polish version. Kardiol Pol. 2009; 67(10): 1166-1169.

11. Fayers PM, Machin D. Quality of life: the assessment, analysis and interpretation of patient-reported outcomes ( $2^{\text {nd }}$ ed.). Wiley, Chichester 2007. 